Immunotherapy is a new and very promising method of anti-cancer treatment. Unfortunately, not every patient can benefit from this treatment. The Polish drug program determines the selection of patients based on PD-L1 expression and the performance status assessed with the use of Eastern Cooperative Oncology Group Performance Status (ECOG PS) score. Patients with ECOG PS 2 represent a significant proportion of the cancer population, one which is overlooked in most clinical trials of immunotherapy. Often, a reduced performance status is the only factor that excludes the patient from treatment with immunotherapy. Choosing the optimal method of treatment in patients with a worse general condition and with multiple diseases may be a significant problem for the doctor. Assessment of performance status may be a particular problem because not every patient has a worse PS score for the same reasons. In this study, we analyse the results of treatment of patients with a poorer performance status to date, and we present tools that improve the precise assessment of the degree of the performance status, which may enable more patients to access novel lung cancer treatments.

Key words: non-small cell lung cancer, immunotherapy, performance status.

Contemp Oncol (Pozn) 2021; 25 (1): 53-56 DOI: https://doi.org/10.5114/wo.2021.105031

\section{Immunotherapy in patients with non-small cell lung cancer with ECOG PS 2}

\author{
Damian Mojsak, Beata Kuklińska, Michał Dębczyński, Robert Marek Mróz
}

Second Department of Lung Diseases and Tuberculosis, Medical University of Białystok, Białystok, Poland

\section{Introduction}

Non-small cell lung cancer (NSCLC) accounts for approximately $85 \%$ of all lung cancer cases. In over $80 \%$ of cases, it is diagnosed at a stage that makes it impossible to use the most effective treatment method, i.e. surgical tumour resection. The results of treatment of patients with advanced disease with the use of classical chemotherapy are unsatisfactory. So far, most patients who did not undergo surgery do not survive the first year after diagnosis. The introduction of molecularly targeted therapy utilizing tyrosine kinase inhibitors has increased the rate of objective responses, improved quality of life, and extended progression-free survival, and in some cases, overall survival (OS). Unfortunately, this treatment was initially only applied to adenocarcinoma patients, large cell, or non-small cell carcinoma without an established histological subtype (NOS).

Recent years have brought new treatment options for patients with nonsmall cell lung cancer. The new drugs have made it possible to improve the quality and extend the life of a larger group of patients, including patients with squamous cell lung cancer.

Immunotherapy is a relatively new and auspicious method of anti-cancer treatment. Immune checkpoint inhibitors (ICls) affect the programmed cell death receptor system type 1 (PD-1 programmed cell death type 1 receptor, PD-L1- programmed cell death receptor ligand). Its own immune system to fight cancer is a key step in cancer control strategy [1].

Reimbursement indications that enable free access to immunotherapy for NSCLC patients in other European Union countries are much more liberal than in Poland. Here, as part of a drug programs reimbursed by the National Health Fund, immunotherapy can be used in patients with stage IV and stage III NSCLC in cases where it is not possible to use radiochemotherapy, radiotherapy, or surgery.

To date, 2 groups of ICls have been registered in the treatment of NSCLC in Poland. The first group, available from 1 May 2018, are anti-PD1 monoclonal antibodies (nivolumab, pembrolizumab) directed against the programmed death receptor. The second group is anti-PD-L1 antibodies directed against the PD1 ligand - the molecule that activates this receptor (atezolizumab), reimbursed since January 2019.

Pembrolizumab is used in the first-line treatment of non-small cell cancer with PD-L1 expression > 50\%. Atezolizumab is approved for the second-line treatment of non-small cell carcinoma patients, both squamous and nonsquamous, regardless of PD-L1 expression. On the other hand, nivolumab can be used in patients with squamous cell lung cancer after failure of prior chemotherapy, regardless of the PD-L1 expression, and from 1 September 2020, it is also approved for use in patients with non-squamous lung cancer.

For the first time in many years of lung cancer treatment, we can see a longterm clinical response in a certain percentage of patients. The use of immunotherapy has significantly extended survival and improved patients' quality of 
life with non-small cell lung cancer [2]. Lung cancer is also increasingly referred to as "chronic neoplastic disease".

However, immunotherapy cannot be used in all patients with non-small cell lung cancer. Drug programs that enable immunotherapy are subject to strict criteria - meeting all of them is necessary to start immunocompetent treatment. A significant problem in everyday practice is patients with an insufficient fitness degree, assessed according to the Eastern Cooperative Oncology Group Performance Status (ECOG PS) scale as grade $\geq 2$. According to the Polish guidelines regarding the $\mathrm{ICl}$ reimbursement program, one of the conditions for qualifying a patient is ECOG PS performance status $\leq 1$. A worse performance status excludes the patient from the possibility of therapy with ICls despite meeting the other qualification criteria.

In this study, we attempt to critically evaluate and optimize the ECOG PS scale criteria, with particular emphasis on the PS 2 patients, which would allow the inclusion of at least some of these patients for immunotherapy. We point out that in many cases, clinical trials excluding this group of patients a priori did not allow justification for the use of immunotherapy. On the other hand, the unsatisfactory treatment outcomes in these patients could have been significantly influenced by the high heterogeneity of these groups and imprecise, subjective assessment of performance status.

\section{Discussion}

In everyday clinical work, doctors often have to face a dilemma regarding the choice of treatment of a patient with non-small cell lung cancer. This group is characterized by vast heterogeneity with patients at various stages of the disease, often with many comorbidities. Before deciding on further actions, it is necessary to assess the patient's general condition, for which we use the ECOG scale, which is required by the $\mathrm{ICl}$ drug program in Poland. This scale is based on the physician's subjective assessment, and at the moment, we do not have entirely objective methods that would allow us to assess the general condition of the patient. Therefore, even doctors in the same centre might assess the performance status differently in the same patient. The subjective assessment of the patient's ECOG PS score by doctors is burdened with an error resulting from the differences in the perception of the general condition of the patient, as well as the diversity of the scales themselves. Therefore, tools are sought that would standardize this assessment. One of them is to replace a one-person assessment with the assessment of at least 2 independent doctors or a conciliar assessment, e.g. of a pulmonologist or oncologist and pulmonary nurse.

A lower degree of performance status may result from comorbidities and the advancement of cancer itself, e.g. due to metastases to the brain, skeleton, or liver, and sometimes from a combination of both of these factors [3]. This fact appears to be particularly important in the case of lung cancer, where smoking is the leading risk factor, which, in addition to increasing the risk of developing lung cancer, also contributes to the development of a wide range of other diseases such as cardiovascular disease, vascular disease, and chronic obstructive pulmonary disease.
It is also worth emphasizing that lung cancer affects middle-aged and older adults more often. About $50 \%$ of cases in both sexes occur in the population over 65 years old [4]. These people often have several extrapulmonary pathologies that affect their degree of efficiency; in this group - reduced physical activity, reduced exposure to air, eating disorders, and more commonly cognitive impairments.

The ECOG scale assesses the degree of fitness due to a combination of all factors that may affect the general condition of the patient. In our opinion, the distinction of whether the deterioration in performance is caused by comorbidities or cancer itself may have a significant impact on the prognosis in the general course of treatment and - perhaps - immunotherapy.

Patients with advanced NSCLC with a lower PS score $(\geq 2)$ according to the ECOG PS scale, cannot receive treatment with ICls, as per the Polish guidelines of the NHF drug program. According to the definition, patients with PS 2 can perform personal activities and are unable to work but remain active for over $50 \%$ of the time during the day [5]. These patients, however, do not constitute a uniform group. Clinical trials, e.g. KEYNOTE and IMpower, which became the basis for the European Medicines Agency (EMA) extension of the indications for the use of immune checkpoint inhibitors, were conducted mainly in patients with ECOG performance level $\leq 1[6,7]$.

Patients with advanced NSCLC with PS $\geq 2$ were most often excluded from clinical trials. Therefore, the response to immune therapy in this group is not fully known. Data on the toxicity and effectiveness of treatment in this group of patients are sparse and do not allow for the unequivocal exclusion of such a procedure in these patients.

In the studies we analysed, the obtained data do not indicate the lack of benefits resulting from immunotherapy in patients with a worse performance degree. Friedlaender et al. [8], after retrospectively analysing the results of treatment of patients with advanced NSCLC with high PD-L1 expression (> 50\%) treated with pembrolizumab in the first line, compared the results of patients with ECOG PS 2 performance with ECOG PS 0-1, revealing that OS and progression-free time (PSF) were almost 3 times lower in patients with ECOG 2; however, due to the lack of a control group, these results do not allow the assessment of treatment benefits in patients with ECOG PS 2 performance status. Importantly, in this study no significant differences in toxicity were observed between the 2 groups.

Ahmed et al. [9], in a retrospective study of 285 patients with NSCLC treated with various levels of immunotherapy (ECOG 0-3), also assessed OSand progression-free survival. The results varied, depending on the ECOG PS score. As expected, the worst results were observed among patients with ECOG performance level 3. Patients with performance level 2 achieved median OS and progression-free survival lower than in patients with ECOG 0 or 1 ; respectively, 8.3 months and 14.7 months (OS) and 5.1 to 7.4 months (PSF). However, despite worse results, these differences did not constitute grounds for disqualifying these patients from this form of treatment.

The CheckMate 171 study assessed the effectiveness of nivolumab treatment in patients with squamous cell 
lung cancer after failure of first-line systemic therapy. This study stands out from other clinical trials because the analysis also included patients with ECOG PS 2. The results of the study confirmed the good tolerance of treatment in this group. It has also been shown that despite a worse response to ECOG 0-1 performance grades, patients with ECOG 2 seem to benefit from their treatment [10].

Conclusions suggesting a beneficial effect of the applied immunotherapy were also put forward by Middleton et al. [11]. After examining a group of 60 NSCLC patients with ECOG PS $=2$ efficiency class qualified for pembrolizumab treatment, they demonstrated the safety of therapy in these patients without increasing the risk of toxicity. Efficacy results were at least as good as in patients with PS 0-1.

It is not easy to qualify a patient to a certain degree of fitness. This was noted in the works of Johansen et al. [12] and Facchinetti et al. [13]. The authors emphasized that assessment of the performance status is a subjective decision of the evaluator. The patient's performance status may result from many factors, e.g. age, reluctance to walk, medications used (opioids, antidepressants), the advancement of the neoplastic disease (emaciation, weakness), and accompanying diseases (e.g. venous insufficiency of the lower limbs). The analysis of both studies' results showed that a better prognosis was obtained in patients in whom the degree of efficiency was determined by the advancement of comorbidities and not the cancer itself.

Facchinetti et al., in a multicentre, retrospective study of patients with NSCLC with PD-L1 expression $\geq 50 \%$ and ECOG performance level 2, who received pembrolizumab in the first line of treatment, showed that such performance status, which results from the existence of comorbidities, is characterized by higher progression-free survival (PFS) and OS than in patients with lower performance status due to the cancer itself [13].

In order to avoid the incorrect disqualification of the patient from immunological treatment and to prevent overlooking important factors that may contribute to the decline in performance status, as well as to enable the prognosis of patients qualified for treatment with ICls, Prelaj et al. [14], DiMaio et al. [15], and Friedlaender et al. [16] indicate the possibility of using scales: EPSILoN (Table 1), DiM (Table 2), or FRAIL (Table 3). These scales are simple and quick tools that help in the efficient as-
Table 1. The EPSILoN scale (Eastern Cooperative Oncology Group Performance Status [ECOG PS], smoking, liver metastases, lactate dehydrogenase [LDH], neutrophil-to-lymphocyte ratio [NLR]) is an acronym named after the factors assessed. This scale is used for prognosis of immunotherapy outcome in the second or further line of treatment

\begin{tabular}{lcc|}
\hline Prognostic factor & Assessment & Points \\
\hline ECOG PS & 1 & 0 \\
& 2 & 1 \\
Smoking (pack years) & $\geq 40$ & 0 \\
& $<40$ & 1 \\
\hline Liver metastases & No & 0 \\
& Yes & 1 \\
LDH (mg/dl) & $<400$ & 0 \\
& $\geq 400$ & 1 \\
NLR & $<4$ & 0 \\
& $\geq 4$ & 1
\end{tabular}

Prognosis: best -0 points, intermediate $-1-2$ points, poor $-3-5$ points

sessment of patients undergoing immunotherapy. Each of these tools takes into account and analyses various factors. Their simultaneous use may be useful in patients for whom the decision to start immunotherapy is difficult, i.e. patients with multiple diseases and with a degree of efficiency that is difficult to establish clearly.

The usefulness of the EPSILoN scale was demonstrated by Prelaj et al. [14]. When assessing the prognostic factors presented on this scale, they found that the median PFS with a favourable result differs by almost 4 months from the unfavourable one, and the difference in OS is over 20 months. The DiM scale was used to determine the prognosis for the response to treatment in the second and subsequent lines of chemotherapy [15] and immunotherapy [16], and it was used by Di Maio et al. [15] and Prelaj et al. [17]. An extensive scale that allows the determination of a favourable or unfavourable prognosis for the analysis utilizes ECOG performance status, gender, histological type of the tumour, tumour stage, previous use of platinum-based chemotherapy, and response to first-line treatment.

The usefulness of the FRAIL ( $F$ - fatigue, $R$ - resistance, A - ambulation, I - illness, $L$-loss of weight) scale in qualifying patients for treatment was assessed by Friedlaender et al. [16]. A quick and straightforward test consisting of

Table 2. DiM scale (Di Maio) was originally used to determine the prognosis of patients undergoing second-line treatment with classical chemotherapy, and it can be used to assess the prognosis of patients treated with immunotherapy. It takes into account the Eastern Cooperative Oncology Group Performance Status (ECOG PS), sex, histological type of the tumour, stage of advancement, previous use of platinum-based chemotherapy, and response to first-line treatment

\begin{tabular}{|c|c|c|c|c|}
\hline \multirow[t]{2}{*}{ Parameter } & \multicolumn{4}{|c|}{ Points } \\
\hline & 0 & 1 & 2 & 7 \\
\hline Sex & Female & Male & - & - \\
\hline ECOG PS & 0 & - & 1 & 2 \\
\hline Tumour stage & III & IV & - & - \\
\hline Histological type & Adenocarcinoma & Squamous & Other & \\
\hline First-line therapy type & Nonplatinum-based & - & Platinum-based & \\
\hline ORR to first line & Yes & No & & \\
\hline
\end{tabular}


Table 3. The FRAIL scale. A scale with 5 simple questions to screen patients for "frailty", which can also be used as a tool to complement the initial assessment before immunotherapy

\begin{tabular}{|c|c|c|}
\hline & Assessment & Question \\
\hline $\mathrm{F}$ & Fatigue & Do you feel tired most or all of the time? \\
\hline $\mathbf{R}$ & Resistance & Can you easily climb the stairs to the first floor? \\
\hline A & Ambulation & Can you walk 1 block without help? \\
\hline I & Illness & Do you have more than 5 comorbidities? \\
\hline L & Loss of weight & $\begin{array}{l}\text { Have you lost > 5\% of your body weight } \\
\text { in the last year? }\end{array}$ \\
\hline
\end{tabular}

several questions (Table 3) can be used as a screening test to select patients for the appropriate oncological treatment. Frailty syndrome is a term that defines a state of reduced physiological reserve resulting in increased susceptibility to stressors such as cancer or aggressive treatment, as well as an increased risk of adverse reactions. The FRAIL scale may be useful in qualifying older patients for immunotherapy; however, its role as a screening tool is not clearly defined.

\section{Conclusions}

Patients with ECOG PS $=2$ constitute a vast, heterogeneous group, including about $40 \%$ of patients with NSCLC [14].

The studies presented above indicate that the performance status assessment should be particularly accurate and individual, allowing the user to distinguish whether the worse degree of efficiency results from the cancer itself and its complications or from the accompanying diseases. The EPSILoN, DiM, and FRAIL scales are easy-to-use tools that can help in the qualification of patients with ECOG PS 2 for immunotherapy.

It seems that the performance status should not separate the patient from immunological treatment. The assessment of the effectiveness of immunotherapy among patients with ECOG PS $\geq 2$ is difficult due to the underrepresentation of this group of patients in clinical trials, who may constitute up to $30 \%$ of patients in real-life conditions.

There is no doubt that the benefit of prolonging OS and PFS is less due to the lower performance status, but this does not imply that immunotherapy is not of significant benefit to these patients. It is necessary to conduct studies taking into account patients with ECOG PS $\geq 2$ in correlation to the factors of in-depth analysis of patients' performance status.

In Poland, ECOG PS 2 is often the only unfulfilled criterion that prevents immunological treatment. Both the US FDA (US Food and Drug Administration) and EMA have registered ICls regardless of the performance status. Drug programs in Poland are successively modified. Since 1 September 2020, nivolumab has been approved for the treatment of patients with not only squamous cell carcinoma, but now also non-squamous lung cancer. Every change in the program for the benefit of the patient is pleasing. Therefore, maybe it is worth at least mitigating the impact of the performance status assessment on the qualification for immunotherapy or going further and eliminating it completely, as in the FDA and EMA.

\section{References}

1. Reck M, Rodríguez-Abreu D, Robinson AG, et al. Pembrolizumab versus Chemotherapy for PD-L1-Positive Non-Small-Cell Lung Cancer. N Engl J Med 2016; 375: 1823-1833.

2. Rebuzzi SE, Leonetti A, Tiseo M, Facchinetti F. Advances in the prediction of long-term effectiveness of immune checkpoint blockers for non-small-cell lung cancer. Immunotherapy 2019; 11: 993-1003.

3. Myers J, Gardiner K, Harris K, et al. Evaluating correlation and interrater relia-bility for four performance scales in the palliative care setting. J Pain Symptom Manag 2010; 39: 250-258.

4. Krajowy Rejestr Nowotworów, Nowotwory złośliwe opłucnej i płuca (C33-34).

5. Oken MM, Creech RH, Davis TE. Toxicology and response criteria of the Eastern Cooperative Oncology Group. Am J Clin Oncol Cancer Clin Trials 1982; 5: 649-655.

6. Mok TSK, Wu Y-L, Kudaba I, et al. Pembrolizumab versus chemotherapy for previously untreated, PD-L1-expressing, locally advanced or metastatic non-small-cell lung cancer (KEYNOTE-042): a randomised, open-label, controlled, phase 3 trial. Lancet 2019; 393: 1819-1830.

7. Cappuzzo F, McCleod M, Hussein M, et al. IMpower 130: efficacy and safety from a randomised phase 3 study of carboplatin and nab-paclitaxel with or without atezolizumab in $1 \mathrm{~L}$ advanced non-squamous NSCLC. ESMO Congress 2018.

8. Friedlaender A, Metro G, Signorelli D, et al. Impact of performance status on non-small-cell lung cancer patients with a PD-L1 tumourproportion score $\geq 50 \%$ treated with front-linepembrolizumab. Acta Oncol 2020; 59: 1058-1063.

9. Ahmed T, Lycan T, Dothard A, et al. Performance status and age as predictors ofimmunotherapy outcomes in advanced none smallcell lung cancer. Clin Lung Cancer 2020; 21: 286-293.

10. Felip E, Ardizzoni A, Ciuleanu T, et al. CheckMate 171: a phase 2 tria of nivolumab in patients with previously treated advanced squamous non-small cell lung cancer, including ECOG PS 2 and elderly populations. Eur J Cancer 2020; 127: 160-172.

11. Middleton G, Brock K, Savage J, et al. Pembrolizumab in patients with non-small-cell lung cancer of performance status 2 (PePS2): a single arm, phase 2 trial. Lancet Respir Med 2020; 8: P895-904.

12. Johansen J, Boisen MK, Mellemgaard A, Holm B. Prognostic value of ECOG performance status in lung cancer assessed by patients and physicians. J Clin Oncol 2013; 31: 8103-8103.

13. Facchinetti F, Mazzaschi G, Barbieri F, et al. First-line pembrolizum$a b$ in advanced non-small cell lung cancer patients with poor performance status. Eur J Cancer 2020; 130: 155-167.

14. Prelaj A, Ferrara R, Rebuzzi SE, et al. Epsilon: a prognostic score for immunotherapy in advanced non-small-cell lung cancer: a validation cohort. Cancers (Basel) 2019; 11: 1954.

15. Di Maio M, Lama N, Morabito A, et al. Clinical assessment of patients with advanced non-small-cell lung cancer eligible for second-line chemotherapy: a prognostic score from individual data of nine randomised trials. Eur J Cancer 2010; 46: 735-743.

16. Friedlaender A, Banna GL, Buffoni L, Addeo A. Poor-performance status assessment of patients with non-small cell lung cancer remains vague and blurred in the immunotherapy era. Lung Cancer 2019; 21: 107.

17. Prelaj A, Lo Russo G, Proto C, et al. DiM: prognostic score for secondor further-line immunotherapy in advanced non-small-cell lung cancer: an external validation. Clin Lung Cancer 2020; 21: e337-e348.

\section{Address for correspondence}

\section{PhD Beata Kuklińska}

Second Department of Lung Diseases and Tuberculosis Medical University of Białystok

14 Żurawia St.

15-540 Białystok, Poland

e-mail: beatakuklinska@op.pl

Submitted: 15.10 .2020

Accepted: 5.11 .2020

The authors declare no conflict of interest. 